\title{
AC 2010-1310: COMPOSITES IN UNDERGRADUATE EDUCATION: AN IMPROVED UNDERSTANDING OF MECHANICS
}

\section{Timothy Hodges, Virginia Military Institute}

Dr. Timothy Hodges is the Professor and Head of Mechanical Engineering at the Virginia Military Institute.

\section{Jon-Michael Hardin, Virginia Military Institute}

Dr. Jon-Michael Hardin is a Professor of Mechanical Engineering at the Virginia Military Insitute. Dr. Hardin received his Ph.D. in Theoretical and Applied Mechanics from the University of Illinois at Urbana-Champaign. 


\section{Composites in Undergraduate Education: An Improved Understanding of Mechanics}

In many cases, students who are learning elementary mechanics of materials are unaware of, or uninterested in, the significance of the assumptions that underlie the elementary mechanics of isotropic materials. However, the significance of those assumptions can be made clear in a study of the mechanics of anisotropic materials. In this paper, the authors will discuss how they effectively highlight those assumptions, and their significance, in a senior-level composite materials course, and how they make use of computer modeling (MathCad) and computer analysis (ANSYS finite-element analysis) within that course to strengthen their students' understanding of both the mechanics of isotropic and anisotropic materials.

\section{$\underline{\text { Mechanics Theory }}^{1,2}$}

The course discussion of mechanics theory used by the authors does not follow the traditional method for the teaching of composite materials, i.e., the mere derivation and simplification of matrices, but rather incorporates student learning of the mechanics of isotropic and anisotropic materials as an integral part of the derivation process itself, through the use of targeted home work assignments and classroom discussions. In addition, the authors have incorporated both scaffolded and reverse-scaffolded learning methods within the targeted homework assignments and the classroom discussions as a unique part of that integrated learning process.

Before the course discussion of the material mechanics, three topics essential for that discussion are presented: 1) tensor notation, 2) the concept of planes of material symmetry, and 3) material types. Tensor notation is typically presented via Figure 1 below to scaffold from the student's knowledge of an orthogonal system of $\mathrm{x}^{-}, \mathrm{y}-$, and $\mathrm{z}$-axis, gained in previous mechanics courses, to the concept of an orthogonal system of 1-, 2-, and 3-axis, along with the stresses that act along those axes.

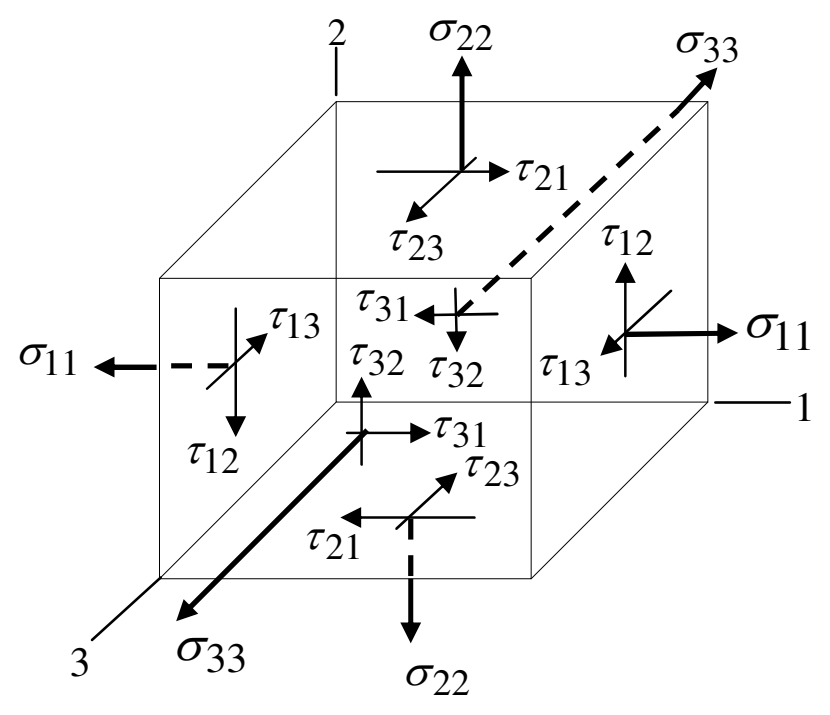

Figure 1 Orthogonal coordinate system and associated normal and sheer stresses 
The concept of a plane of material symmetry is a more complex concept for students to understand. Typically, the following definition is provided initially:

A plane is called a plane of material symmetry when the elastic constants at a point within the material remain unchanged for every pair of coordinate systems that are mirror images of each other within that plane.

Students are then led through a discussion of the application of this definition to an isotropic material. Through this discussion, students gain a greater appreciation for the meaning of the definition of a plane of material symmetry and a new understanding that isotropic materials as a group are uniquely defined by the fact that they all have an infinite number of planes of material symmetry.

This discussion of isotropic materials leads naturally to a discussion of other types of materials that do not have infinite numbers of planes of material symmetry: anisotropic materials that have no planes of material symmetry and orthotropic materials that have 3 mutually orthogonal planes of material symmetry. Although the students were told in their previous elementary mechanics of materials class that the relationships developed in that course were valid only for isotropic materials, they gain a better initial appreciation for that restrictive assumption by merely discussing the existence of anisotropic and orthotropic materials and by being questioned whether they believe those elementary mechanics relationships would be valid also for these other types of materials. Since most students intuitively feel that the elementary mechanics relationships would not hold for non-isotropic materials, they become curious about the relationships that would be valid for those materials.

The discussion of those relationships begins with the introduction of the generalized Hooke's Law

$$
\sigma_{i j}=C_{i j k l} \varepsilon_{k l}
$$

that, the students are surprised to find, represents nine stresses and nine strains related by 81 theoretically independent elastic constants. The discussion of this relationship is scaffolded from the student's prior knowledge of Hooke's Law for an isotropic material loaded in pure tension

$$
\sigma=E \varepsilon
$$

for an isotropic material loaded in pure torsion

$$
\tau=G \gamma
$$

and for an isotropic material under general three-dimensional loading

$$
\sigma_{x}=\frac{E}{(1+v)(1-2 v)}\left[(1-v) \varepsilon_{x}+v\left(\varepsilon_{y}+\varepsilon_{z}\right)\right] \text { or } \quad \varepsilon_{x}=\frac{\sigma_{x}}{E}-\frac{v}{E}\left(\sigma_{y}+\sigma_{z}\right)
$$




$$
\begin{gathered}
\sigma_{y}=\frac{E}{(1+v)(1-2 v)}\left[(1-v) \varepsilon_{y}+v\left(\varepsilon_{x}+\varepsilon_{z}\right)\right] \text { or } \varepsilon_{y}=\frac{\sigma_{y}}{E}-\frac{v}{E}\left(\sigma_{x}+\sigma_{z}\right), \\
\sigma_{z}=\frac{E}{(1+v)(1-2 v)}\left[(1-v) \varepsilon_{z}+v\left(\varepsilon_{x}+\varepsilon_{y}\right)\right] \text { or } \varepsilon_{z}=\frac{\sigma_{z}}{E}-\frac{v}{E}\left(\sigma_{x}+\sigma_{y}\right), \text { and } \\
\tau_{x y}=G \gamma_{x y}, \tau_{y z}=G \gamma_{y z}, \tau_{x z}=G \gamma_{x z}
\end{gathered}
$$

Students are reminded of the fact that only two of the three elastic constants $E, G$, and $v$ are independent since

$$
G=\frac{E}{2(1+v)} .
$$

As a part of a homework exercise (Homework 1), students are asked to write the above equations for stress in matrix form and identify each element of the $[C]$ matrix:

$$
\left\{\begin{array}{l}
\sigma_{x} \\
\sigma_{y} \\
\sigma_{z} \\
\tau_{x y} \\
\tau_{y z} \\
\tau_{x z}
\end{array}\right\}=[C]\left\{\begin{array}{l}
\varepsilon_{x} \\
\varepsilon_{y} \\
\varepsilon_{z} \\
\gamma_{x y} \\
\gamma_{y z} \\
\gamma_{x z}
\end{array}\right\} .
$$

\section{(Homework 1)}

Students are then questioned as to why/how the 81 theoretically independent elastic constants contained in the generalized Hooke's Law might collapse to merely 2 independent elastic constants in the case of an isotropic material. They are now primed to follow the trail of assumptions that lead to that collapse.

The first assumption discussed is the symmetry in $\sigma_{i j}$ and $\varepsilon_{j l}$, i.e.,

$$
\sigma_{i j}=\sigma_{j i} \text { and } \varepsilon_{k l}=\varepsilon_{l k} \quad \text { (Assumption 1) }
$$

which reduces the number of stresses and of strains from 9 to 6 and which causes $C_{i j k l}=C_{j i k l}$ and $C_{i j k l}=C_{i j l k}$, thus reducing the number of independent elastic constants from 81 to 36 . Important to this discussion is the question of whether this assumption holds for all types of materials from anisotropic to isotropic. As a homework exercise (Homework 2), students are asked to show, using the results from their Homework 1 assignment, that Assumption 1 
is valid and that $C_{i j k l}=C_{j i k l}$ is true for an isotropic material.

The second assumption discussed is the symmetry in $C_{m n}$ such that

$$
C_{m n}=C_{n m}
$$

(Assumption 2)

where the following changes of subscripts is made from $i j$ and $k l$ to $m$ and $n$

$$
\begin{gathered}
11(i j \text { or } k l)->1(m \text { or } n) \\
22->2 \\
33->3 \\
23->4 \\
13->5 \\
12->6
\end{gathered}
$$

results in the relationship

$$
\sigma_{m}=C_{m n} \varepsilon_{n}
$$

Assumption 2 reduces the number of independent elastic constants from 36 to 21. Again, students are asked whether this assumption is valid for all material types from anisotropic to isotropic. In a related homework assignment (Homework 3) students are asked to

1) list of the specific 15 dependent relationships represented by Assumption 2 for the general case (e.g., $C_{1122}=C_{2211}, C_{2313}=C_{1323}$ ),

2) write these $[C]$ matrix elements in matrix from, and

3) show that Assumption 2 holds for an isotropic material based upon the elements of the $[C]$ matrix developed in their Homework 1 assignment.

The third discussed assumption (Assumption 3) results from the fact that an orthotropic material has three mutually orthotropic planes of material symmetry and so

\section{(Assumption 3)}

$$
\begin{aligned}
& C_{14}=C_{15}=C_{16}=0 \Rightarrow \text { (by Assumption 2) } C_{41}=C_{51}=C_{61}=0 \\
& C_{24}=C_{25}=C_{26}=0 \Rightarrow \text { (by Assumption 2) } C_{42}=C_{52}=C_{62}=0 \\
& C_{34}=C_{35}=C_{36}=0 \Rightarrow \text { (by Assumption 2) } C_{43}=C_{53}=C_{63}=0 \\
& C_{45}=C_{46}=0 \quad \quad \Rightarrow>(\text { by Assumption } 2) \quad C_{54}=C_{64}=0 \\
& C_{56}=0 \quad \Rightarrow>\left(\text { by Assumption 2) } C_{65}=0,\right.
\end{aligned}
$$

thereby reducing the number of independent elastic constants from 21 to 9 , where 


$$
\begin{gathered}
C_{11}=E_{1} \frac{1-v_{23} v_{32}}{\Delta}, \quad C_{22}=E_{2} \frac{1-v_{13} v_{31}}{\Delta}, C_{33}=E_{3} \frac{1-v_{12} v_{21}}{\Delta}, \\
C_{12}=E_{1} \frac{v_{21}+v_{31} v_{23}}{\Delta}=E_{2} \frac{v_{12}+v_{32} v_{13}}{\Delta}, \\
C_{13}=E_{1} \frac{v_{31}+v_{21} v_{32}}{\Delta}=E_{3} \frac{v_{13}+v_{12} v_{23}}{\Delta}, \\
C_{23}=E_{2} \frac{v_{32}+v_{12} v_{31}}{\Delta}=E_{3} \frac{v_{23}+v_{21} v_{13}}{\Delta}, \text { and } \\
C_{44}=G_{23} ; C_{55}=G_{13} ; C_{66}=G_{12}
\end{gathered}
$$

and

$$
\Delta=1-v_{12} v_{21}-v_{23} v_{32}-v_{31} v_{13}-2 v_{21} v_{32} v_{13}
$$

Assumption 3 is discussed in-depth with the students since it is not valid for general anisotropic materials, unlike assumptions 1 and 2, but is valid for orthotropic and isotropic materials, and since it forms the basis for much of the lamina mechanics relationships in fiber-reinforced composite materials. As part of a homework assignment (Homework 4), students are asked to compare these [C] matrix elements with the [C] matrix elements for isotropic materials that they developed in Homework 1.

The final assumption (Assumption 4) discussed is for isotropic materials, that is, those materials that have an infinite number of planes of material symmetry. For this assumption

$$
\begin{gathered}
E_{1}=E_{2}=E_{3}=E, \\
G_{13}=G_{23}=G_{12}=G, \text { and } \\
v_{13}=v_{23}=v_{12}=v_{31}=v_{32}=v_{21}=v
\end{gathered}
$$

(Assumption 4)

This assumption results in the number of elastic constants being reduced from 9 to 2 so that

$$
C_{11}=C_{22}=C_{33}=\lambda+2 \mu, C_{12}=C_{13}=C_{23}=\lambda, \text { and } C_{44}=C_{55}=C_{66}=\mu
$$

where $\quad \lambda=\frac{E v}{(1+v)(1-2 v)}$ and $\mu=G=\frac{E}{2(1+v)}$.

As part of a homework assignment (Homework 5) the students are asked to compare the above [C] elements with the [C] elements they developed in Homework 1.

Through the use of scaffolding from previously gained knowledge about isotropic materials students are better prepared to develop new knowledge about the mechanics of both anisotropic and orthotropic materials. However, a type of reverse-scaffolding process also occurs by which the students gain a deeper understanding of the assumptions that restrict the use of elementary 
mechanics concepts to isotropic materials only. This scaffolding/reverse-scaffolding process is reinforced through the use of MathCad and ANSYS to model and analyze isotropic, transversely isotopic, and orthotropic composite materials. The students have access to the MathCad and ANSYS programs through departmental site licensing and acquired the ability to use these programs in previous, pre-requisite courses. Through the use of these programs, students can easily and quickly determine and 'see' the effects of changes in material (isotropic, transversely isotropic, and orthotropic) or loading.

\section{Computer Modeling and Analysis}

The lamina mechanics of fiber-reinforced composite materials taught in this course are primarily for the case of plane stress. Therefore, all of the following discussions of computer modeling and analysis are limited to that case.

\section{Computer Modeling using $\mathrm{MathCad}^{3}$}

MathCAD, a very powerful computational software package, was selected for use in this course because of its ease of programming and its ability to closely resemble the mathematical expressions in written form. This feature allows the student to concentrate on the engineering without being overwhelmed by the programming. The students use MathCad to develop, over the course of the semester, a computer model that includes both the lamina and laminate mechanics of a fiber-reinforced material. Also, in composite material mechanics, the $[\mathrm{C}]$ matrix is typically denoted as the [Q] stiffness matrix for loading that coincides with the fiber direction (i.e., $0^{\circ}$ angle) and is denoted as $[\bar{Q}]$ for loading at a non-zero angle with respect to the fiber direction.

The first example modeled by the students in this course is for an isotropic material in loaded in plane stress at $\mathrm{a}^{\circ}$ angle where the material properties are given as E11 $:=207 \cdot \mathrm{GPa}, \mathrm{E} 22:=$ $207 \cdot \mathrm{GPa}, v 12:=0.33$ and $G_{12}:=\frac{E_{11}}{2 *\left(1+v_{12}\right)}=77.82 \mathrm{GPa}$. Using these material property values, the students determine the values of the $[\mathrm{Q}]$ matrix elements:

$$
\left[Q_{i s o}\right]=\left[\begin{array}{ccc}
232.297 & 76.658 & 0 \\
76.658 & 232.297 & 0 \\
0 & 0 & 77.82
\end{array}\right] G P a
$$

To demonstrate the directional-independence of the isotropic case, the students also determine the $\left[\bar{Q}_{i s o}\right]$ matrix element values for an angle of $30^{\circ}$ from the load direction by using the relationships

$$
\begin{gathered}
\bar{Q}_{11}=Q_{11} \cos ^{4} \theta+2\left(Q_{12}+2 Q_{66}\right) \sin ^{2} \theta \cos ^{2} \theta+Q_{22} \sin ^{4} \theta \\
\bar{Q}_{22}=Q_{22} \cos ^{4} \theta+2\left(Q_{12}+2 Q_{66}\right) \sin ^{2} \theta \cos ^{2} \theta+Q_{11} \sin ^{4} \theta \\
\bar{Q}_{66}=\left(Q_{11}+Q_{22}-2 Q_{12}-2 Q_{66}\right) \sin ^{2} \theta \cos ^{2} \theta+Q_{66}\left(\sin ^{4} \theta+\cos ^{4} \theta\right)
\end{gathered}
$$




$$
\begin{gathered}
\bar{Q}_{12}=\left(Q_{11}+Q_{22}-4 Q_{66}\right) \sin ^{2} \theta \cos ^{2} \theta+Q_{12}\left(\sin ^{4} \theta+\cos ^{4} \theta\right) \\
\bar{Q}_{16}=\left(Q_{11}-Q_{12}-2 Q_{66}\right) \sin \theta \cos ^{3} \theta+\left(Q_{12}-Q_{22}+2 Q_{66}\right) \sin ^{3} \theta \cos \theta \\
\bar{Q}_{26}=\left(Q_{11}-Q_{12}-2 Q_{66}\right) \sin ^{3} \theta \cos \theta+\left(Q_{12}-Q_{22}+2 Q_{66}\right) \sin \theta \cos ^{3} \theta .
\end{gathered}
$$

or the case of a $30^{\circ}$ angle, the students can see that the $\left[\bar{Q}_{\boldsymbol{i s o} \boldsymbol{o} 0^{\circ}}\right\rfloor$ matrix element values remain unchanged from those found in the $\left[Q_{i s o}\right]$, zero-degree case for an isotropic material. This comparison of values, obtained from the orthotropic mechanics relationships for fiber-reinforced materials, re-enforces for the students the uniqueness of the isotropic case within the broader case of orthotropic materials.

In the second MathCad model, students determine the $[Q]$ stiffness matrix values for a fiberreinforced material for $0^{\circ}$ angle of loading (i.e., for a transversely isotropic material) using the given material properties of $\mathrm{E} 11:=207 \cdot \mathrm{GPa}, \mathrm{E} 22:=19 \cdot \mathrm{GPa}, v 12:=0.21$, and G12 $:=6.4 \cdot \mathrm{GPa}$ (note that the E11 value used in this example is the same as that used in the earlier isotropic example.) Using the given material properties the students determine that

$$
\left[Q_{\text {transiso }}\right]=\left[\begin{array}{ccc}
207.841 & 4.006 & 0 \\
4.006 & 19.077 & 0 \\
0 & 0 & 6.4
\end{array}\right] G P a \text {. }
$$

Comparing these values to those determined for the purely isotropic $0^{\circ}$ angle case, the students become aware that the difference in the value of E22 between these two cases tremendously affects the values of the transverse Q12 element as well as the Q22 and Q16 elements.

Using the same material property values the students also determine the values of the stiffness matrix for the case of loading at an angle of $30^{\circ}$ to the fiber direction to be:

$$
\left[\bar{Q}_{30}^{\circ}\right]=\left[\begin{array}{ccc}
124.405 & 40.251 & 61.795 \\
40.251 & 30.023 & 19.943 \\
61.795 & 19.943 & 42.645
\end{array}\right] G P a
$$

It is obviously apparent to the students that the elements of this matrix are vastly different from those found for the $0^{\circ}$ angle case. Most importantly, the non-zero $\bar{Q}_{16}$ and $\bar{Q}_{16}$ element results found in this example lead naturally to a discussion of the normal-shear stress-strain coupling found in composite materials that does not exist in isotropic materials.

In addition to comparing the stiffness matrix element values determined for each of the cases noted above, the students are also asked to compare those values to the assumptions discussed in the Theory portion of the course to see if those assumptions indeed hold in these cases. 
From these comparisons of the stiffness matrix values of the isotropic cases and the orthotropic cases, students gain a much better understanding of the unique behavioral character of isotropic materials, especially the lack of possibility of normal-shear stress-strain coupling regardless of loading angle. This unique characteristic of isotropic materials is further reinforced through an ANSYS analysis.

\section{Computer Analysis using ANSYS ${ }^{4}$}

An ANSYS analysis of an isotropic material and a fiber-reinforced material under a similar loading provides a dramatic visual representation of the differences in behavior between these two types of materials. Using a thin plate, the ANSYS results show an outline of the original unloaded shape of the plate and superimpose on that image the deformed shape after loading.

The first analysis conducted by the students is for an isotropic material loaded with $\mathrm{P}$ in the $\mathrm{y}$ direction and with $2 \mathrm{P}$ in the $\mathrm{x}$-direction. The results of the analysis are shown in Figure 2 below, along with a superposition of the load.

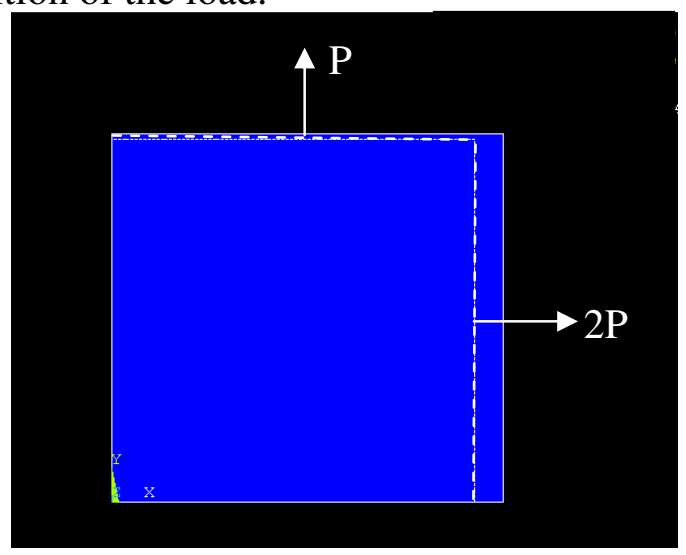

Figure 2 ANSYS analysis of an isotropic material

This result shows that the greater loading in the $\mathrm{x}$-direction produces a greater deformation in that direction. There is also clearly no normal-shear stress-strain coupling.

The second analysis is for a fiber-reinforced composite material similarly loaded at an angle of $0^{\circ}$ to the fiber direction (i.e., transversely orthotropic).

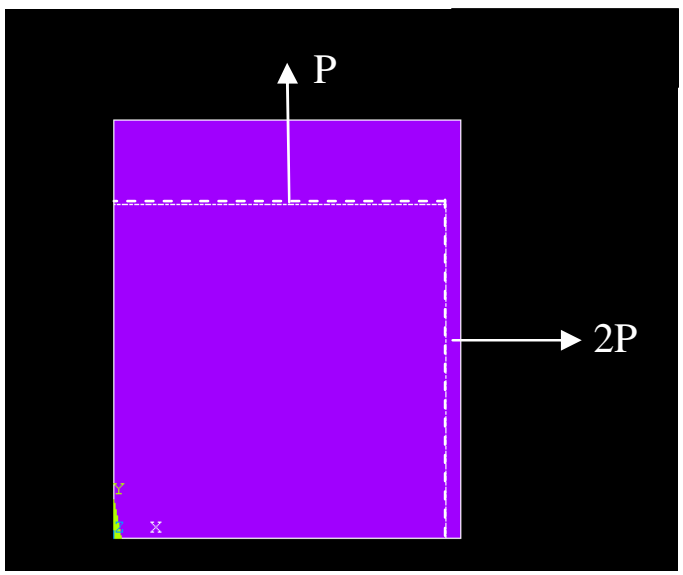

Figure 3 ANSYS analysis of $0^{\circ}$ loading of a fiber-reinforced composite material 
Students can easily see from this result how the fiber reinforcement of the matrix material along the $\mathrm{x}$-direction decreases the deformation in that direction. However, there is a larger deformation in the y-direction because there are no fibers in the y-direction and so the deformation in that direction is due primarily to the matrix material properties. This deformation contrasts sharply with the deformation seen in the isotropic case.

The third analysis the students conduct is for a fiber reinforced material loaded at an angle of $30^{\circ}$ to the fiber direction.

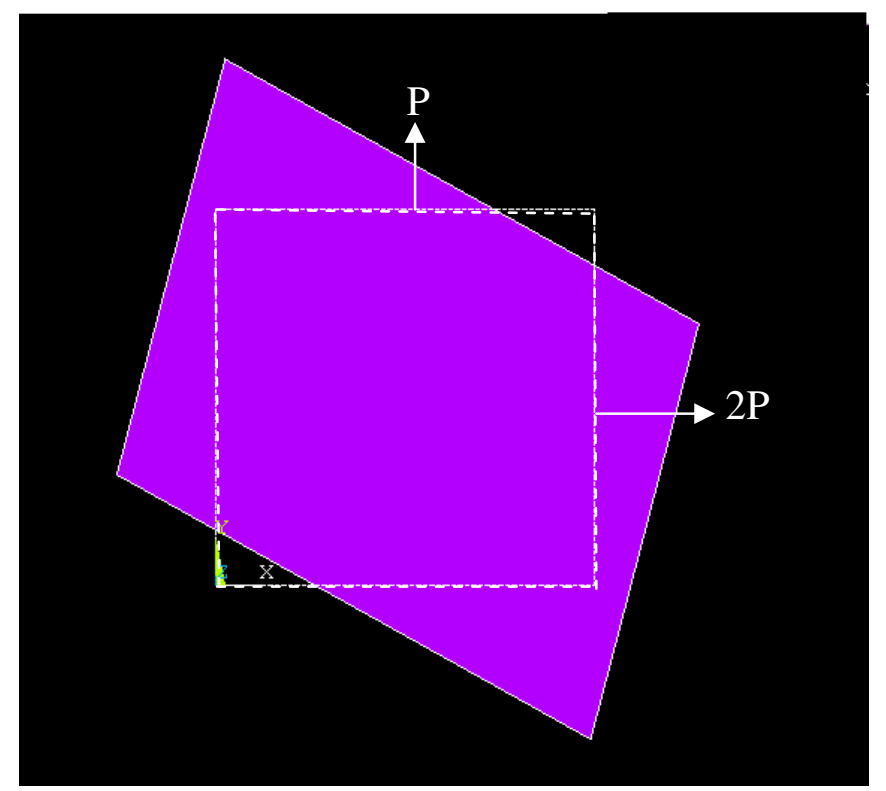

Figure 4 ANSYS analysis of $30^{\circ}$ loading of a fiber-reinforced composite material

Although there is no applied shear load, the student can see clearly from this analysis the normalshear coupling that the student would not expect to see based on their study of isotropic materials. This dramatic visualization of the normal-shear coupling effect helps students to better understand both the complex behavior of composite materials and the simplicity of the behavior of isotropic materials.

\section{$\underline{\text { Conclusion }}$}

Due to the small number of students enrolled in the annual one section offering of this composites course, the authors have been unable to conduct a meaningful statistical study to determine the efficacy of their methods. They hope to conduct such a study, in the future, through a combined effort with other universities. However, based on informal focus group discussions with their students and on their own extensive teaching experience in both elementary solid mechanics and composite materials, they believe that, by combining a theoretical discussion of the assumptions that underlie the mechanics of anisotropic, orthotropic, and isotropic materials with targeted computer modeling and analysis of both isotropic and orthotropic materials, student understanding of the unique character of both isotropic materials and orthotropic composite materials can be greatly strengthened. 


\section{Bibliography}

1. Reddy, J.N. Mechanics of Laminated Composite Plates, CRC Press, Boca Raton, FL, 1997,

2. Jones, M.R. Mechanics of Composite Materials, $2^{\text {nd }}$ Edition, Taylor and Francis, Inc., Philadelphia, PA, 1999.

3. Larsen, R.W. Introduction to Mathcad 13, Pearson Prentice Hall, Upper Saddle River, NJ, 2007.

4. ANSYS Verification Manual Input Listings, ANSYS Release 10, ANSYS, Inc., Canonsburg, PA, 2005. 